\title{
Transmission of Images Over Noisy Channels Using Error-Resilient Wavelet Coding and Forward Error Correction
}

\author{
Nikolaos V. Boulgouris, Nikolaos Thomos, Student Member, IEEE, and Michael G. Strintzis, Senior Member, IEEE
}

\begin{abstract}
A novel embedded wavelet coding scheme is proposed for the transmission of images over unreliable channels. The proposed scheme is based on the partitioning of information into a number of layers which can be decoded independently provided that some important and highly protected information is initially errorlessly transmitted to the decoder. Forward error correction is used in conjunction with the error-resilient source coder for the protection of the compressed stream. Unlike many other robust coding schemes presented to date, the proposed scheme is able to decode portions of the bitstream even after the occurrence of uncorrectable errors. This coding strategy is very suitable for application with block coding schemes such as defined by the JPEG2000 standard. The proposed scheme is compared with other robust image coders and is shown to be very suitable for transmission of images over memoryless channels.
\end{abstract}

Index Terms-Channel coding, error resilience, Viterbi algorithm, wavelets.

\section{INTRODUCTION}

$\mathbf{M}$ ANY new techniques have been recently proposed for the efficient coding of images [2]-[4] and video [5], [6]. However, the transmission of the pictorial information over today's heterogeneous, and often unreliable, networks (as in broadcasting applications) has necessitated the provision of protection methods against possible channel failures. Although, in theory, source and channel coding can be studied independently (Shannon's separation principle [7]), channel coding strategies which take into consideration the structure of the underlying source coder produce significantly better performance [8].

A variety of coders based on error correcting codes have been proposed in the literature. Sherwood and Zeger [9] divide the bitstream output by the popular SPIHT coder [2] into blocks of constant length. Each packet is protected by a concatenated rate-compatible punctured convolutional code and cyclic redundancy check code (RCPC/CRC). In a subsequent paper [10], Sherwood and Zeger propose using product codes consisting of

Manuscript received May 14, 2001; revised June 11, 2003. This work was supported by the EU IST projects "ASPIS" and "OTELO." This work appeared in part in the Proceedings of the IEEE International Conference of Image Processing. This paper was recommended by Associate Editor O. K. Al-Shaykh.

N. V. Boulgouris was with the Informatics and Telematics Institute, 57001 Thermi, Thessaloniki, Greece. He is now with the Electrical and Computer Engineering Department, University of Toronto, Toronto, ON, Canada.

N. Thomos and M. G. Strintzis are with the Information Processing Laboratory, Electrical and Computer Engineering Deptartment, Aristotle University of Thessaloniki, Thessaloniki 54124 , Greece. They are also with the Informatics and Telematics Institute, 57001 Thermi, Thessaloniki, Greece (e-mail: strintzi@eng.auth.gr).

Digital Object Identifier 10.1109/TCSVT.2003.819187 convolutional codes and Reed-Solomon (RS) codes in order to protect the SPIHT source stream more efficiently over memoryless and fading channels. In the aforementioned techniques, the first uncorrectable error causes loss of synchronization between encoder and decoder, due to the use of an arithmetic coder, and the decoding process is terminated.

Man et al. [11] introduce two methods for coding the location information of significant subband coefficients. The output bitstream is protected by applying RCPC channel codes. Tanabe and Farvardin [12] propose two different wavelet-based schemes, the first using differential pulse code modulation (DPCM) and the second two-dimensional (2-D) discrete cosine transform (DCT) coding for the coding of the lowest frequency band. The other subbands are quantized using zero-memory quantizers, whose output is entropy coded and protected using RCPC codes. In [13], transmission of images over lossy packet networks was studied and an unequal error protection strategy was followed, optimized using Lagrange multipliers.

Chande and Farvardin [14] proposed a bit allocation algorithm for application with embedded coders. They applied their scheme with the SPIHT source coder. Mohr et al. [15] also investigated the transmission of images over lossy packet networks using ideas initially explored in [16]. A scheme for robust transmission of SPIHT streams was also studied in [17]. Finally, Banister et al. [18] proposed a scheme for the protection of JPEG2000 streams [19] using turbo-codes.

In all of the aforementioned algorithms, decoding of the received robust streams stops at the first uncorrectable error. This has the obvious drawback of losing a potentially high portion of the bitstream (i.e., all bits following the first uncorrectable error). This situation deteriorates dramatically with noisier channels, since then the first uncorrectable error may occur very early in the stream.

In this paper, we first design an error-resilient source coder which is also very suitable for use in joint source/channel coding systems. The proposed scheme employs wavelet decomposition and exhibits lossy performance similar to that of the state-of-the-art SPIHT coder. It is based on the partitioning of information into a number of layers which can be decoded independently provided that some very important and highly protected information is initially errorlessly transmitted to the decoder. The independent bitstreams are subsequently protected using equal or unequal amounts of protection. Forward error correction (FEC) based on RCPC codes is used. This coding approach allows the decoding of the bitstream even after the occurrence of uncorrectable errors and thus differentiates our 
scheme from other zerotree-based or block-based robust coders seen so far in the literature.

Robust coders based on the error-resilient source coding framework are implemented and evaluated. Specifically, two variants of the source coder employing rate-distortion optimized and nonoptimized transmission of layers are used. The tradeoff between layer size and side information is also evaluated for transmission over two different channel conditions. The behavior of the new coders is tested in case of channel mismatch and is shown to operate much more efficiently in comparison to SPIHT-based robust image coders.

The contribution of the present paper is:

- an error-resilient source coding scheme;

- a novel blockwise source/channel coding strategy which clearly distinguishes significance and refinement layers [3] during the rate allocation process;

- an evaluation of robust image coding with respect to parameters such as the rate-distortion optimized transmission or the number of layers in which coding has been performed.

The organization of the paper is as follows. In Section II, the novel source coder specifically designed to serve robust image transmission applications is described. In Section III, unequal error protection (UEP) is investigated for use with the proposed source coder. The efficient detection and handling of errors not corrected by the channel code is discussed in Section IV. In Section V, a bit allocation algorithm is presented. Experimental evaluation is presented in Section VI, and, finally, conclusions are drawn in Section VII.

\section{A Simple Scalable IMAge Coder}

\section{A. Independent Layer Coding}

The proposed wavelet image coding scheme is based on the following coding techniques:

- tree-structure exploitation;

- bitplane coding of subbands;

- block classification;

- conditional arithmetic coding of wavelet coefficients.

After the multiresolution decomposition of the image, the lowest frequency coefficients are decorrelated using DPCM, coded using arithmetic coding and placed in the image header. Tree structures $\mathcal{T}$ are formed which are comprised of coefficients that lie in the subbands that have the same orientation across scales. Each tree structure has a root. Each root has $2 \times$ 2 children and each child has $2 \times 2$ children and so on until the highest resolution subband is reached. For each of these tree structures, the maximum absolute value of the coefficient is found and the number of bits (typically 1-7) which are required for its representation is placed in the image header

$$
\max =\left\lfloor\log _{2} \max _{(i, j) \in \mathcal{T}}\{c(i, j)\}\right\rfloor
$$

where $c(i, j)$ is the absolute value of the wavelet coefficient in position $(i, j)$. In addition, the maximum absolute coefficient in each subband is also placed in the image header. All tree and subband maxima are arithmetically encoded.
The subbands in the two highest resolution levels of the image are further split into blocks of $32 \times 32$ pixels. Blocks in each subband in the two highest resolution levels are grouped in two classes according to their energy and each class is coded independently from the others. Hereafter, the term "block" may mean a block or a group of blocks. The transmission of information for each block is done in a bitplane-wise manner starting from the most significant bit to the least significant bit. For each block, first the coefficients whose most significant bit lie in the bitplane currently coded are identified by comparing them to a threshold $T=2^{n}$ where $n=0, \ldots, N$, is the index of the bitplane that is being coded and $N$ is the maximum bitplane index. If a coefficient becomes significant (i.e., it is found to be greater than or equal to $T$ for the first time) then its sign is coded. This process is often called significance identification [20] and the layer including this information will be hereafter denoted as $\mathcal{L}_{n k}^{s}$ where $k$ is the block index. Similarly, the refinement layer, defined as the one containing the $n_{\text {th }}$ bitplane of coefficients found significant in previous passes, will be denoted as $\mathcal{L}_{n k}^{r}$.

The $n_{\mathrm{th}}$ bit in the binary representation of a coefficient in tree $\mathcal{T}$ and subband $\mathcal{B}$ is coded if and only if both requirements listed below are valid:

1) The maximum coefficient in tree $\mathcal{T}$ is greater than or equal to the current threshold

$$
\max _{(i, j) \in \mathcal{T}}(c(i, j)) \geq 2^{n}
$$

2) The maximum coefficient in the subband $\mathcal{B}$ is greater than or equal to the current threshold

$$
\max _{(i, j) \in \mathcal{B}}(c(i, j)) \geq 2^{n} .
$$

The deployment of the above rules drastically reduces the number of coefficients whose significance is tested during the coding of a significance identification layer. However, in order to further reduce the number of symbols that have to be coded during the layer coding stage, a single bit is initially coded to indicate if all coefficients in a block are insignificant.

The symbol streams described above are coded using adaptive arithmetic codes [21]. The context modeling strategy in [3] is followed for the coding of significance identification layers. Refinement bits are entropy coded using a single adaptive arithmetic model. The max_frequency_count of the arithmetic coder was set equal to 512 in order to allow fast adaptation of the coder to the statistics of the incoming symbol stream.

Bitplane encoding is practically equivalent to quantizing wavelet coefficients with successively finer quantizers whose quantization stepsize is $2^{n}$ when the $n$th bitplane is being coded. The reconstructed value for such coefficients would be in the middle of the quantization bin. However, in order to further enhance the efficiency of the source coding scheme, we calculate the reconstruction value $2^{n}+q_{n}$ for a coefficient refined up to bit $n$ as the average of the absolute values of coefficients within the interval $2^{n}$ and $2^{n+1}$. The reconstruction levels are calculated for each decomposition level and are quantized to 3-b accuracy. These values are stored in the compressed file. This approach yields a gain of approximately $0.15 \mathrm{~dB}$. An alternative, and possibly more efficient, approach 
where reconstruction levels can be computed using modeling of wavelet coefficients can be found in [22]. However, the method described above is simpler.

\section{B. Transmission of Layers}

1) Rate-Distortion Optimized Transmission Order: The order in which the block layers $\mathcal{L}_{n k}^{s}, \mathcal{L}_{n k}^{r}$ coded as above, will be transmitted, is determined on the basis of their distortion reduction capability [3], [23]. Specifically, if the distortion decrease caused by the $n_{\text {th }}$ layer of the $k_{\text {th }}$ block, whose coding requires $R_{n k}$ bits, is defined by

$$
D_{n k}=w^{2} \sum_{i}\left(\left(\hat{c}_{i}^{n+1}-c_{i}\right)^{2}-\left(\hat{c}_{i}^{n}-c_{i}\right)^{2}\right)
$$

where $c$ and $\hat{c}$ denote the original and the reconstructed wavelet coefficients, then block bitplanes for which the ratio $D_{n k} / R n_{k}$ is large are coded first. The weighting parameter $w$ (which is different for each subband) is needed because wavelet data in different subbands contribute to the quality of the reconstructed image in different ways. Actually, this parameter is intended to compensate for the nonorthogonality of the filter bank and is computed as in [24].

The distortion decrease that is caused by the identification of $N$ significant coefficients when the $n_{\text {th }}$ most significant bitplane is coded is given by [23]

$$
D_{n k}=w^{2} 2.25 T_{n}^{2} N
$$

where $T_{n}=2^{n}$. Trivially, for refinement coding, the distortion decrease achieved when $N_{1}$ coefficients are refined to the $n_{\text {th }}$ bit can be approximated by

$D_{n k}=w^{2} T_{n}^{2}\left(1+t_{n}-2 t_{n+1}\right)^{2} N_{r 1}+w^{2} T_{n}^{2}\left(t_{n}-2 t_{n+1}\right)^{2} N_{r 0}$

where $t_{n}=q_{n} / T_{n}$ and $N_{r 0}, N_{r 1}$ are, respectively, the numbers of zeros and ones in the refinement layer.

The resulting coding scheme will be referred to as scalable wavelet image coding (SWIC). Using (2) and (3), layers are ordered based on their distortion decrease capability and optimally progressive transmission is achieved. However, when seen from a coding point of view, this comes at the cost of having to transmit a layer identifier in the header of each layer. A different method, similar in some respects to that used for integer wavelets in [25], may be defined so as to use a classical predefined scanning order, which obviates the need for the transmission of layer identifiers. This method will be described and evaluated next.

2) Filter-Dependent Transmission Order: Instead of using optimized transmission of layers, we can alternatively transmit bitplanes of subbands without regard to their exact distortion decrease capability (although no blocks are used here, if each subband is seen as an individual block, the terminology of the previous subsection is still valid; actually, this case is a special case of the previous one, where all blocks are equal to subbands and the transmission order is predefined). This approach yielded coders with very good performance in [25] and [26], where integer wavelets were used. In this case, subband bitplanes are transmitted in a predefined order which depends only on the

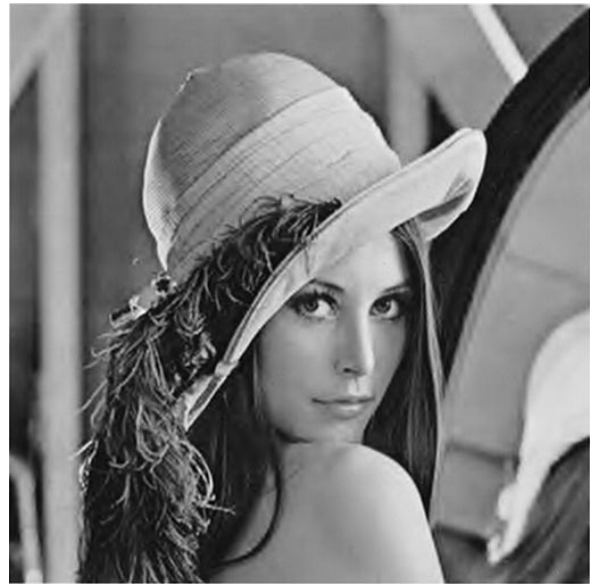

(a)

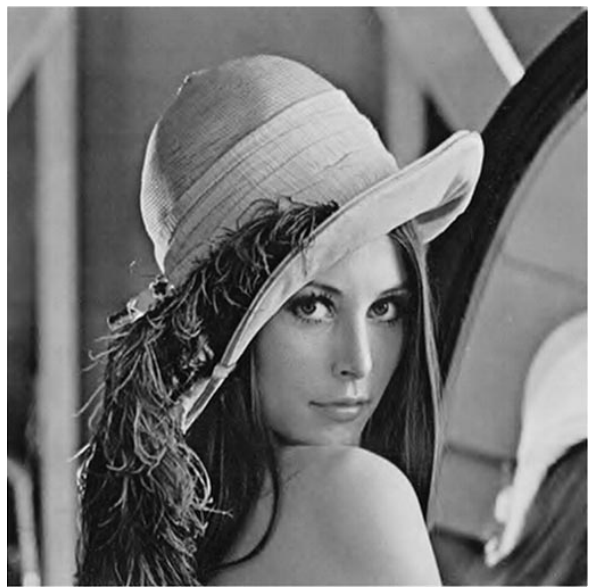

(b)

Fig. 1. Reconstructed "Lenna" using only SWIC coding (noiseless): (a) 0.25 b/pixel (33.88 dB) and (b) $0.5 \mathrm{~b} /$ pixel $(37.07 \mathrm{~dB})$.

filter used for the decomposition [24]. Our coder actually is one of the simplest possible bitplane coders since in order to maintain an error-resilient bitstream organization we do not resort to complex context modeling or fractional bitplanes, as other coders do.

This approach has the disadvantage of disregarding the fact that information within the same subband is unevenly distributed; however, it has the advantage that layer identifiers are not required. The latter is particularly useful in robust coding applications such as those studied in this paper. The coding scheme described above will be referred to as predefined-order scalable wavelet image coding (PSWIC).

In the experimental results section, both techniques, rate-distortion optimized (SWIC) and nonoptimized (PSWIC), are evaluated and conclusions are drawn about their application to image transmission over unreliable channels.

\section{Performance of the Source Coder}

After recovering a wavelet representation of the transmitted image, the decoder applies the inverse wavelet transform used for the reconstruction of the initial image. Reconstructed images using the SWIC coder are shown in Fig. 1. For the results in this paper, the image is decomposed using the popular Daubechies 9/7 bi-orthogonal wavelet filters. Five levels of decomposition 
TABLE I

Comparison of the Proposed Source Coding Schemes WitH STATE-OF-THE-ART CODERS. RECONSTRUCTION PSNR IN dB

\begin{tabular}{c|c|c|c|c}
\hline bitrate & SWIC & PSWIC & SPIHT & EBCOT \\
\hline \hline 0.0625 & 27.85 & 27.98 & 28.39 & 28.10 \\
\hline 0.125 & 30.83 & 30.94 & 31.10 & 31.05 \\
\hline 0.25 & 33.88 & 33.96 & 34.11 & 34.16 \\
\hline 0.5 & 37.07 & 36.95 & 37.21 & 37.29 \\
\hline 0.75 & 39.15 & 39.17 & 39.04 & N/A \\
\hline 1.00 & 40.34 & 40.17 & 40.44 & 40.48 \\
\hline
\end{tabular}

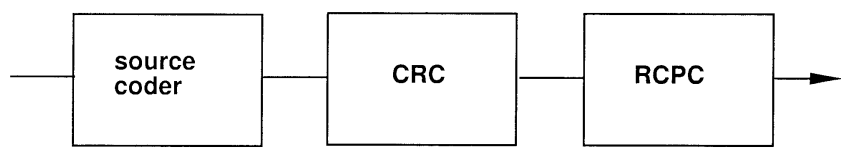

Fig. 2. Operations for the efficient protection of layers.

are used. The lossy performance of the proposed SWIC and the PSWIC schemes in comparison to the set partitioning in hierarchical trees (SPIHT) coder with arithmetic coding and the embedded block coding with optimized truncation (EBCOT) coder [3] is reported in Table I. As seen, the proposed source coding schemes have approximately the same performance as that of SPIHT. Application of more complicated techniques, such as the fractional bitplanes described in [23] or the optimized truncation described in [3], could further improve the performance of the source coder. While these further source coding optimizations will not be presented in this paper, initial investigations suggest that they could be added without diminishing the robustness over noisy channels.

\section{PRotection of COMPRESSED StREAMS}

The layers produced as described in the previous section are coded using channel coding [27]. Since each bitplane of a block is coded without using information from other blocks, protection can be individually applied to each such block. A schematic description of the system used for the generation of robust streams is shown in Fig. 2.

Specifically, header information, i.e., dc coefficients and tree and subband maxima, are considered very important information and are highly protected. An error in the header would be catastrophic and would render the rest of the stream useless. We should note, however, that header information represents a tiny portion of the compressed image and thus the additional protection needed to ensure its uncorrupted transmission is affordable, since it introduces negligible redundancy in terms of bits per pixel. For the results in the present paper, the header of the test image was protected using rate-16/23 codes.

Layers $\mathcal{L}_{n k}^{s}$ and $\mathcal{L}_{n k}^{r}$ corresponding to significance identification and refinement coding are channel coded. The basic structure for adding protection is depicted in Figs. 3 and 4. Each layer is independently protected by employing a field in its header which states the size of the source bits used for the coding of that layer. Another field in the header specifies the matrix with which the RCPC codes are punctured [27]. This is very useful in cases where a whole layer has to be discarded (due to uncorrectable errors) since the length of the source+channel rate of the layer can be deduced at the decoder side and, thus, the corrupted layer can be discarded without preventing subsequent layers (that do not depend on the discarded layer) from being decoded correctly (see Fig. 3).

For the efficient protection of layers, each layer $\mathcal{L}$ is partitioned into $N_{p}(\mathcal{L})$ packets of equal size (apart from the last packet, which may be shorter) and protected using the coder shown in Fig. 2. This is shown in Fig. 4. Note that the (nonconstant) size of the last packet in a layer can be implicitly calculated from the size of the layer and the puncturing matrix identifier (which are stored in the layer header). Thus, no other side information is needed for its coding and decoding.

\section{EFFICIENT ERROR HANDLING}

A significant feature of a robust coder is its ability to detect and confine errors not corrected by the channel code. Zerotree-based coders are not suitable for error-resilient image transmission since the occurrence of a single erroneous bit renders the rest of the bitstream undecodable. In such coders, if an error is not detected, then the quality of the reconstructed image will be totally unacceptable. In our coder, due to the bitstream generation and organization strategy followed, errors not corrected by the channel code affect usually only the packet in which the error occurred and occasionally a few subsequent packets.

For the detection of errors, cyclic redundancy codes (CRC) [28] are employed in conjunction with RCPC codes [27]. For the efficient correction of errors, the serial list Viterbi algorithm [29] was used with a list of 100 paths. When the list Viterbi algorithm is used, the optimal path in the Viterbi decoding is chosen among those paths that follow the constraints imposed by the CRC [10].

Alternately, in the very rare case where an uncorrectable error is not detected by the CRC check, the detection of errors can be performed using another mechanism. Since each layer is decoded independently and the size of the layer is a priori known to the decoder, the event of an uncorrectable error can be easily detected due to loss in the arithmetic code resynchronization. This means that the arithmetic decoder attempts to decode symbols beyond the anticipated end of the layer, disclosing the existence of an uncorrected error. This technique bears some resemblance to the scheme proposed in [30] where a redundant symbol whose arithmetic decoding reveals to be erroneous stimulates a repeat request (ARQ). This scheme however, assumes the availability of a feedback channel via which ARQ signs can be transmitted to the transmitter. Our approach is different in the sense that the proposed error-resilient framework obviates the need for redundant symbols or ARQ signals and does not require a feedback channel. The reader who is interested in gaining additional insight to issues concerning the resynchronization properties of arithmetic codes is referred to [31].

The detection of an uncorrected error during decoding stimulates the following actions.

- If the error is in layer $\mathcal{L}_{n k}^{s}$, then this layer is retained up to the first corrupted packet and all subsequent layers $\mathcal{L}_{j m}^{s}$, 


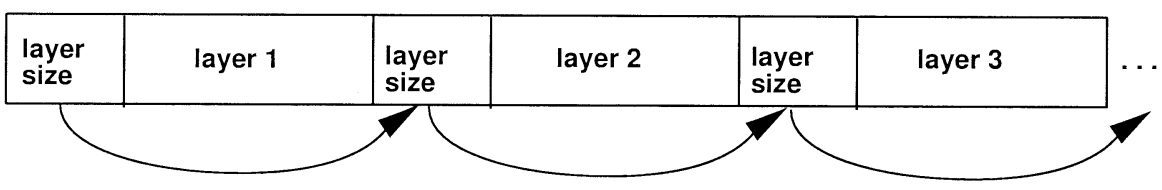

Fig. 3. Bitstream structure. The beginning of each layer is a highly protected header indicating the size of the layer. If an uncorrected error occurs in a layer, the corrupted layer can be discarded and the decoding process can proceed with the next uncorrupted layer.

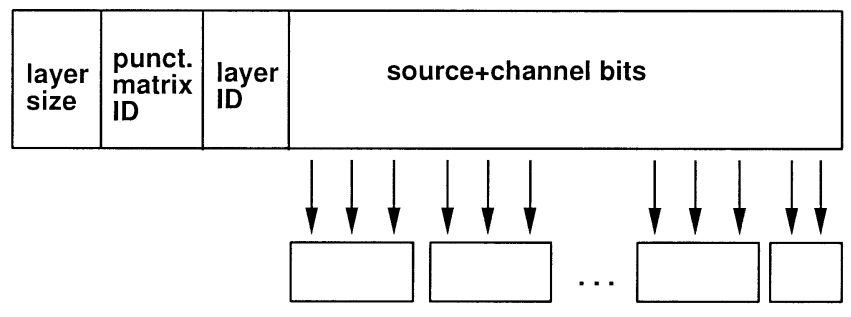

Fig. 4. Organization of information in a robust layer. If the rate-distortion optimized transmission scheme is used, the layer identifier is needed. Otherwise, it is not required. The layer is divided into packets of equal size (apart from the last packet) and each packet is protected separately.

$\mathcal{L}_{j m}^{r}, j<n, m=k$, for the same block are discarded since the information they contain can not be exploited. This process is illustrated in Fig. 5.

- If the error is in $\mathcal{L}_{n k}^{r}$, then this layer is retained up to the first corrupted packet. The rest of the packets comprising the layer are discarded, but all subsequent layers $\mathcal{L}_{j k}^{r}, j<$ $n$, are retained (provided that no uncorrectable error occur in those layers) since such errors are localized and do not affect the decoding of subsequent layers.

The ability of our robust coding methodology to discard corrupted portions of the bitstream in order to confine errors and achieve the best possible reconstruction quality endows the proposed scheme with the capability of achieving superior performance. This will be shown in the experimental results section where a family of robust coders, built using the techniques described so far, are evaluated. The allocation of protection to the source stream will be examined in the ensuing section.

\section{BlockWise UNEQUAL ERror Protection}

Since information bits are coded in order of importance, unequal error protection can be naturally incorporated in our coder by consistently allocating more bits to layers transmitted first and fewer channel bits to subsequent layers. An algorithm for the optimal allocation of protection will be described in the present section. For the sake of ease of reference a short table is included (Table II), explaining the notation used in the remainder of the section.

In order to allocate bits between source and channel, we first note that each additional portion of the bitstream that is made available to the decoder reduces the distortion between the original and the reconstructed image. Thus, the problem can be described as that of maximization of the distortion decrease $D$ achieved when bitplanes from $Q(k)$ to $N(k)$ for block $k$ are to be transmitted

$$
D=\sum_{k=1}^{M} D_{k}=\sum_{k=1}^{M} \sum_{i=Q(k)}^{N(k)}\left(D^{s}(i, k)+D^{r}(i, k)\right)
$$

subject to a total rate constraint $R_{T}$. $D_{k}$ is the distortion decrease for block $k, M$ is the number of blocks of wavelet coefficients (some blocks may be as large as an entire subband), $N(k)$ is the maximum nonzero bitplane index in the $k_{\mathrm{th}}$ block, and $D^{s}(n, k), D^{r}(n, k)$ are the cumulative distortion reductions achieved by the transmission of bitplanes $n, \ldots, N(k)$ of the $k_{\text {th }}$ block for significance and refinement layers, respectively. Finally, $Q(k)$ is the bitplane (determined in a way to be discussed later in this section) at which transmission stops for each block $k .{ }^{1}$ All notation is summarized in Table II in which the superscripts ${ }^{s}$ and ${ }^{r}$, aiming to distinguish between significance and refinement layers, are dropped from symbols referring to layer quantities. Note that, for each block, layers with higher bitplane indices are transmitted before layers with lower bitplane indices. Therefore, for each block, transmission begins with the layer indexed $N(k)$ and continues to $N(k)-1, \ldots, n$. The average distortion decrease caused by the transmission of significance layers $n, \ldots, N(k)$ for the $k_{\text {th }}$ block is

$$
D^{s}(n, k)=(1-P(n, k)) \sum_{m=n}^{N(k)} D_{m k}^{s}
$$

where $D_{n k}^{s}$ denotes the individual distortion decrease caused by layer $\mathcal{L}_{n k}^{s}$ and $1-P(n, k)$ denotes the probability that only layers $\mathcal{L}_{m k}^{s}, m=n, \ldots, N(k)$, are correctly decoded, i.e., the probability that the first corrupted layer in the $k_{\mathrm{th}}$ block is $\mathcal{L}_{(n-1) k}^{s}$. Since the decoding of a layer is possible only if all previous (more significant) layers have been decoded correctly, this probability is equal to

$$
1-P(n, k)=P_{(n-1) k}^{s}\left(r_{(n-1) k}^{s}\right) \prod_{m=n}^{N(k)}\left(1-P_{m k}^{s}\left(r_{m k}^{s}\right)\right)
$$

where $P_{n k}^{s}\left(r_{n k}^{s}\right)$ is the individual probability that a significant layer is not decoded correctly (i.e., supposing that all layers it depends on are correctly decoded) when $r_{n k}^{s}$ is the channel code rate used for its coding. Similarly, the distortion decrease caused by refinement layers $n, \ldots, N(k)$ for the $k_{\mathrm{th}}$ block is

$$
\begin{aligned}
D^{r}(n, k)= & P_{(n-1) k}^{s}\left(r_{(n-1) k}^{s}\right) \prod_{m=n}^{N(k)}\left(1-P_{m k}^{s}\left(r_{m k}^{s}\right)\right) \\
& \times\left(\sum_{m=n}^{N(k)}\left(1-P_{n k}^{r}\left(r_{n k}^{r}\right)\right) D_{m k}^{r}\right)
\end{aligned}
$$

where $D_{n k}^{r}$ now denotes the individual distortion decrease caused by layer $\mathcal{L}_{n k}^{r}$ and $P_{n k}^{r}\left(r_{n k}^{r}\right)$ is the individual probability that a refinement layer is not decoded correctly. In practice, the

${ }^{1}$ We assume that refinement layers for the $k_{\mathrm{th}}$ block are transmitted up to the $Q(k)$ bitplane for all transmitted significance layers. This assumption is not very restrictive since refinement layers are much shorter than their corresponding significance layers. 


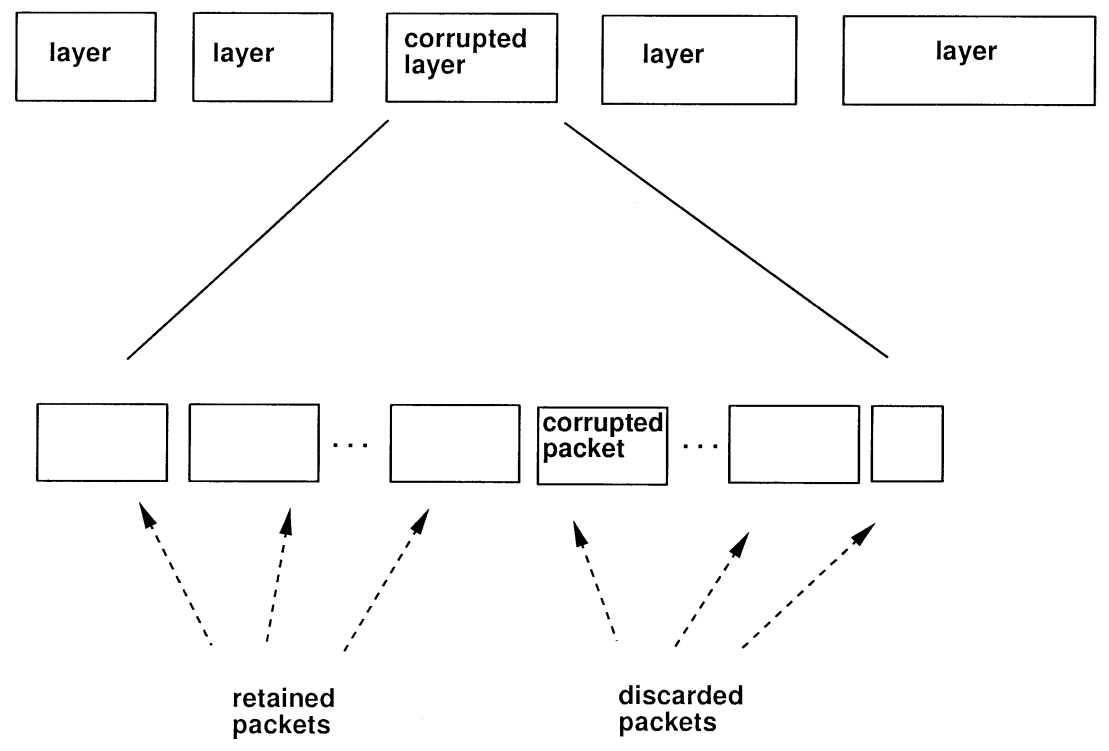

Fig. 5. Packet disposal as performed by the proposed coder in case of uncorrectable errors.

TABLE II

NOTATION

\begin{tabular}{c|c}
\hline Symbol & Notation \\
\hline \hline$k$ & Block index \\
$n$ & Bitplane index \\
$N(k)$ & Max bitplane index for block $k$ \\
$Q(k)$ & Min bitplane index for block $k$ \\
$D_{k}$ & Distortion decrease for block $k$ \\
$R_{k}$ & Channel rate for block $k$ \\
$D_{n k}$ & Distortion decrease for layer $\mathcal{L}_{n k}$ \\
$R_{n k}$ & Source rate for layer $\mathcal{L}_{n k}$ \\
$r_{n k}$ & Channel code rate for layer $\mathcal{L}_{n k}$ \\
$P_{n k}\left(r_{n k}\right)$ & Corrupted layer probability \\
\hline
\end{tabular}

above equation disregards the fact that uncorrectable errors in a refinement layer may affect the distortion reduction capability of subsequent refinement layers. However, this approach yields a simpler formulation.

Each layer $\mathcal{L}_{n k}$ is divided into $N_{p}\left(\mathcal{L}_{n k}\right)$ constant-length packets and each packet is individually protected. The probability that a layer is discarded is equal to the probability that at least one packet in this layer is plagued by uncorrectable errors. If $p$ is the probability that a packet is corrupted, then the probability of $l$ corrupted packets among the $N_{p}\left(\mathcal{L}_{n k}\right)$ packets that comprise a layer $\mathcal{L}_{n k}$ coded using channel code rate $r_{n k}$ is

$$
P\left(l, N_{p}\left(\mathcal{L}_{n k}\right), r_{n k}\right)=\left(\begin{array}{c}
N_{p}\left(\mathcal{L}_{n k}\right) \\
l
\end{array}\right) p^{l}(1-p)^{N_{p}\left(\mathcal{L}_{n k}\right)-l}
$$

and, therefore, the probability of a layer error (of the existence of at least one packet in the layer in error) is given by the expression

$$
P_{n k}\left(r_{n k}\right)=\sum_{l=1}^{N_{p}\left(\mathcal{L}_{n k}\right)} P\left(l, N_{p}\left(\mathcal{L}_{n k}\right), r_{n k}\right) .
$$

Since the probability of an uncorrectable packet depends on the RCPC code used, this probability is experimentally evaluated for the set of channel codes used.

As seen from (6), refinement layers $\mathcal{L}_{n k}^{r}$ depend only on previous significant layers $\mathcal{L}_{j k}^{s}, j \geq n$, in the same block. Essentially, (6) expresses the probability that a layer $\mathcal{L}_{n k}$ is not decoded due to errors in previous layers $\mathcal{L}_{j k}, n<j \leq N(k)$. Using (5)-(7), (4) becomes

$$
\begin{aligned}
D= & \sum_{k=1}^{M} \sum_{i=Q(k)}^{N(k)} P_{(i-1) k}^{s}\left(r_{(i-1) k}^{s}\right) \\
& \times\left(\sum_{n=1}^{N(k)}\left(D_{n k}^{s}+\left(1-P_{n k}^{r}\left(r_{n k}^{r}\right)\right) D_{n k}^{r}\right)\right) \\
& \times \prod_{n=i}^{N(k)}\left(1-P_{n k}^{s}\left(r_{n k}^{s}\right)\right)
\end{aligned}
$$

where $P_{(Q(k)-1) k}^{s}\left(r_{(Q(k)-1) k}\right)=1$ and $D_{N(k) k}^{r}=0$. The optimization problem then becomes that of maximizing the distortion decrease $D$ given by (10) subject to the total available channel rate $R_{T}$. The transmitted rate $R$ can be expressed as

$$
R=\sum_{k=1}^{M} R_{k}=\sum_{k=1}^{M} \sum_{i=Q(k)}^{N(k)}\left(\frac{R_{i k}^{s}}{r_{i k}^{s}}+\frac{R_{i k}^{r}}{r_{i k}^{r}}\right)
$$

where $R_{n k}^{s}\left(R_{n k}^{r}\right)$ denotes the source bits used for the coding of $\mathcal{L}_{n k}^{s}\left(\mathcal{L}_{n k}^{r}\right)$.

The allocation of channel bits to source layers can be facilitated by the following.

Theorem 1 [32]: In block-based "independent allocation" coding strategies, i.e., if the rate and distortion can be measured independently for each block, quantization is Rate-Distortion (R-D) optimal if it yields identical R-D slopes for all code blocks.

Theorem 2: For the R-D optimal protection of R-D optimized source streams, the channel code rate should be such that information is equally protected. Thus, if $D_{i} / R_{i}$, is the partial 
R-D slope for the $i_{\mathrm{th}}$ channel-coded block, then $D_{i} / R_{i}=c$, for all blocks $i=1, \ldots, M$.

Proof: Optimal channel coding means that unequal amounts of protection are allocated to the various portions of the source bitstream according to their importance. However, from Theorem 1, the slopes corresponding to source-coded blocks are constant, i.e., the information transmitted for each block is equally important. Thus, after channel coding the slopes corresponding to the channel-coded blocks should be equal too.

Theorem 3: Consider a channel rate allocation $\left\{\left(D_{1}, R_{1}\right)\right.$, $\left.\left(D_{2}, R_{2}\right), \ldots,\left(D_{M}, R_{M}\right)\right\}$. The overall R-D slope

$$
\lambda=\frac{D_{1}+D_{2}+\ldots+D_{M}}{R_{1}+R_{2}+\ldots+R_{M}}
$$

is maximized if $D_{i} / R_{i}$ is maximized for each block independently.

Proof: Let the optimal rate allocation be $\left\{\left(D_{i}, R_{i}\right)\right\}$, $i=1, \ldots, M$ and another arbitrary one be $\left\{\left(D_{i}^{\prime}, R_{i}^{\prime}\right)\right\}$, $i=1, \ldots, M$. Then

$$
\begin{aligned}
\lambda^{\prime} & =\frac{D_{1}^{\prime}+D_{2}^{\prime}+\ldots+D_{M}^{\prime}}{R_{1}^{\prime}+R_{2}^{\prime}+\ldots+R_{M}^{\prime}} \\
& =\frac{R_{1}^{\prime} \frac{D_{1}^{\prime}}{R_{1}^{\prime}}+R_{2}^{\prime} \frac{D_{2}^{\prime}}{R_{2}^{\prime}}+\ldots+R_{M}^{\prime} \frac{D_{M}^{\prime}}{R_{M}^{\prime}}}{R_{1}^{\prime}+R_{2}^{\prime}+\ldots+R_{M}^{\prime}} \\
& <\frac{R_{1}^{\prime} \frac{D_{1}}{R_{1}}+R_{2}^{\prime} \frac{D_{2}}{R_{2}}+\ldots+R_{M}^{\prime} \frac{D_{M}}{R_{M}}}{R_{1}^{\prime}+R_{2}^{\prime}+\ldots+R_{M}^{\prime}}
\end{aligned}
$$

where the initial assumption $\left(D_{i} / R_{i}\right)>\left(D_{i}^{\prime} / R_{i}^{\prime}\right)$ was used. But, from Theorem 2, we have

$$
\frac{D_{i}}{R_{i}}=c .
$$

Thus, (12) yields

$\lambda^{\prime}=\frac{D_{1}^{\prime}+D_{2}^{\prime}+\ldots+D_{M}^{\prime}}{R_{1}^{\prime}+R_{2}^{\prime}+\ldots R_{M}^{\prime}}<c \cdot \frac{R_{1}^{\prime}+R_{2}^{\prime}+\ldots+R_{M}^{\prime}}{R_{1}^{\prime}+R_{2}^{\prime}+\ldots R_{M}^{\prime}}=c$.

However,

$$
\begin{aligned}
\lambda & =\frac{D_{1}+D_{2}+\ldots+D_{M}}{R_{1}+R_{2}+\ldots R_{M}} \\
& =\frac{R_{1} \frac{D_{1}}{R_{1}}+R_{2} \frac{D_{2}}{R_{2}}+\ldots+R_{M} \frac{D_{M}}{R_{M}}}{R_{1}+R_{2}+\ldots+R_{M}}=c .
\end{aligned}
$$

The theorem follows.

Although the conclusion (Theorem 1) of optimality of equal slopes corresponding to source-coded blocks is not strictly valid in practical embedded coders, such as those presented in this paper, experimental evaluation demonstrates that channel rate allocation based on this assumption yields good results. Specifically, Theorem 3 above shows that optimal unequal error protection using a block-based source coding scheme, such as the one presented in this paper, can be achieved by optimizing the allocation on a blockwise basis.

Blockwise optimization of rate allocation is possible if the optimal source and channel rates for each block are known. In practice, this can be achieved using the techniques in [33], by solution of an unconstrained problem which aims to the maximization of an objective function $F$ of the form

$$
F=D-\lambda_{L} R
$$

where $D$ and $R$ are given by (10) and (11), respectively, and $\lambda_{L}$ is a Lagrange multiplier. Provided the channel conditions are known, the error probability $P_{n k}$ can be calculated for each layer and the determination of $Q(k)$ and $r_{n k}$ for $k=1, \ldots, M$ is achieved via the maximization of (15) for the appropriate $\lambda_{L}$. However, the optimal determination of $Q(k)$ and $r_{n k}$ involves iterative solution of (15), i.e., iterative calculation of $Q(k)$ and $r_{n k}$, as $\lambda_{L}$ converges to its optimal value $\lambda_{L}^{*}$ [33]. This may be a computationally demanding procedure.

In this work, in order to determine the appropriate rates for each block, we take a simpler approach by assuming that the channel code rate which is applied for the protection of the entire stream and the individual blocks is approximately equal to the code rate used by an equal error protection (EEP) scheme. Since the channel bit error rate (BER) is known, the channel code rate of the EEP scheme is determined by calculating (10) for all available channel code rates and selecting the code rate yielding the largest distortion reduction as the most appropriate for use in an EEP scheme. Based on this assumption, the transmitted source layers can be deduced and, therefore, the terminating source bitplane and the channel rate, $Q(k)$ and $R_{k}$, respectively, are determined for each block. Optimal selection of the code rates $r_{n k}$ is then possible using exhaustive search or dynamic programming techniques. After computing $r_{n k}$ for each layer, then the corresponding RCPC code is applied. The channel bit allocation proceeds for all subsequent blocks and the corresponding allocations are determined. Since in practice only a limited number of possible code rates is available, the solution is not really optimal. However, in most cases the available code rates are sufficient for achieving high-performance transmission.

The proposed joint source/channel coding algorithm is summarized below.

1) Compute the wavelet transform of the image.

2) Partition the wavelet representation into blocks.

3) Encode each bitplane $n$ of each block $k$ of coefficients into two layers: $\mathcal{L}_{n k}^{s}$ and $\mathcal{L}_{n k}^{r}$ corresponding to the significance and refinement pass, respectively.

4) Order the layers using one of the two techniques described in Section II.

5) Find the best set of channel code rates for each layer using the algorithm in this section.

6) Encode each layer with the appropriate channel code.

It should be noted that, in channel bit allocation techniques, the rate allocation complexity increases significantly with the number of source blocks in which rate is allocated (see, e.g., [14], [18]). In order to reduce the computational load introduced by the optimization process, a small number of large source blocks may be used. However, this makes rate control more difficult (this is an important drawback in embedded coding since large blocks do not offer a fine range of available rates). On the other hand, if a large number of small source blocks is used, the computational complexity of the optimization process increases dramatically. For this reason, our blockwise methodology (which divides the optimization problem in a number of individually treated optimizations) appears to be very suitable 
since it enjoys the best from both aforementioned approaches without further increasing complexity.

\section{EXPERIMENTAL EVALUATION}

The proposed coders were experimentally evaluated for image transmission over binary symmetric channels (BSCs). The $512 \times 512$ "Lenna" image was used in the simulations. Comparison was based on the average quality of the reconstructed image for two channel conditions. Specifically, two BSCs were simulated with BER $=0.01$ and 0.001 , respectively.

The CRC codes used were taken from [34]. The family of RCPC codes that was used is based on a rate- $1 / 4$, memory- 6 mother code given by the generator tap matrix

$$
\mathrm{g}=\left\{g_{i k}\right\}=\left[\begin{array}{ccccccc}
1 & 1 & 0 & 1 & 1 & 0 & 1 \\
1 & 0 & 1 & 0 & 0 & 1 & 1 \\
1 & 0 & 1 & 1 & 1 & 1 & 1 \\
1 & 1 & 0 & 0 & 1 & 1 & 1
\end{array}\right]
$$

in which a 1 in line $i$, column $k$, represents a connection from the $k_{\text {th }}$ shift register stage to the $i_{\text {th }}$ output. The convolutional encoder corresponding to the above generator matrix is depicted in Fig. 6. The output of the encoder was punctured (i.e., certain code bits were not transmitted) using the puncturing matrices determined by the allocation process of Section V. The puncturing matrices change the code rate and hence the correction power of the code according to source and channel needs. Eight puncturing matrices (see Appendix I) were employed with rates $\{16 / 17,8 / 9,16 / 19,8 / 10,16 / 21,8 / 11,16 / 23,8 / 12\}$. In most practical applications, for $\mathrm{BER} \leq 10^{-2}$, puncturing with the above matrices is sufficient. Extending the set of available matrices would yield vanishingly negligible gain since the more appropriate protection would be outbalanced by the increase in the cost for the transmission of matrix indices. It is noted that the total channel code rates used by our coders, i.e., the ratios of the source bits to the channel bits, are slightly higher than the channel code rates used by the other methods in our comparisons. The source rate saved by the rate allocation procedure partly outbalances the performance difference between our source coder and SPIHT.

Three robust coders were implemented and included in the comparisons.

- SWIC: The SWIC algorithm of Section II-B-1 channel coded in two different ways: in a single layer and in multiple layers.

- PSWIC: the PSWIC algorithm of Section II-B-2 channel coded by coding significance and refinement information independently (as different layers) using a predefined scan order of subbands and bitplanes.

- HSWIC: A hybrid coder which begins as PSWIC (the order of the 50 initial layers, approximately up to $0.1 \mathrm{bpp}$ source rate, is predefined) and continues as SWIC (the order of the rest of the layers is R-D optimized). This scheme has the advantage of avoiding the transmission of layer headers for a large number of short layers which are usually seen in the beginning of the embedded stream.

The algorithms compared to the present coders in terms of average reconstruction quality were those by Sherwood [9], Man [11], and Chande [14]. The methods in [9], [11], and [14] all em-

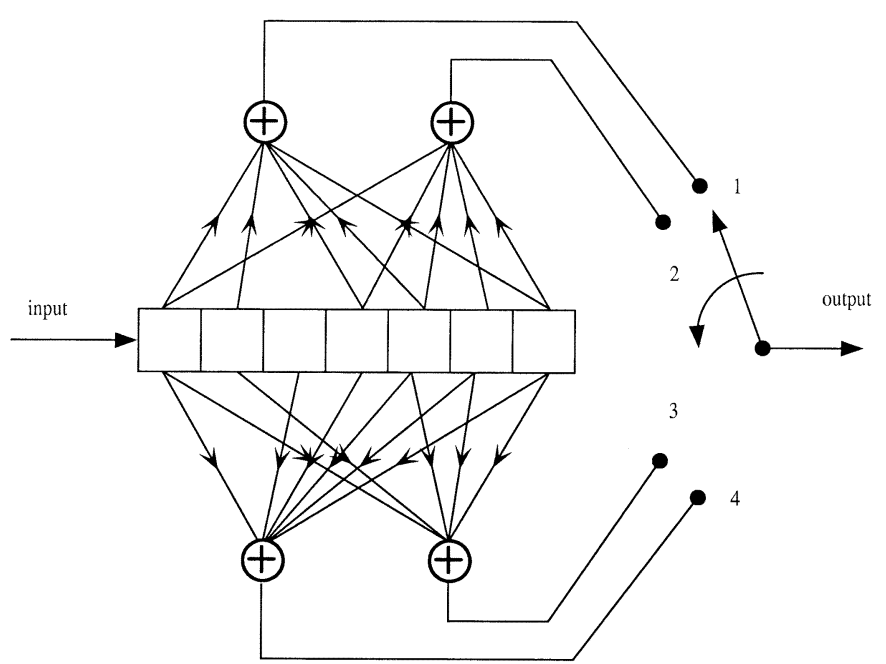

Fig. 6. Memory-6 convolutional encoder.

TABLE III

COMPARISON OF THE PROPOSED CODING SCHEME FOR THE TRANSMISSION OF IMAGES OVER BSC WITH BER $=0.01$. EEP AND UEP WAS USED WITH THE PROPOSED SCHEMES

\begin{tabular}{c|c|c|c|c|c|c|c|c}
\hline \multirow{2}{*}{} & \multicolumn{2}{|c|}{ SWIC } & \multicolumn{2}{c|}{ PSWIC } & \multicolumn{2}{c|}{ HSWIC } & Sherwood [9] & Chande [14] \\
\cline { 2 - 8 } & single & multiple & EEP & UEP & EEP & UEP & EEP & UEP \\
\hline 0.25 & 32.17 & 31.75 & 32.16 & $\mathbf{3 2 . 3 0}$ & 32.01 & 32.10 & 31.91 & $\mathbf{3 2 . 3 0}$ \\
\hline 0.5 & 35.29 & 35.01 & 35.25 & $\mathbf{3 5 . 3 9}$ & 35.09 & 35.18 & 34.96 & 35.28 \\
\hline 1.00 & 38.24 & 38.11 & 38.31 & $\mathbf{3 8 . 4 1}$ & 38.17 & 38.25 & 38.03 & 38.28 \\
\hline
\end{tabular}

TABLE IV

COMPARISON OF THE PROPOSED CODING SCHEMES FOR THE TRANSMISSION OF IMAGES OVER BSC WITH BER $=0.001$

\begin{tabular}{c|c|c|c|c|c}
\hline & $\begin{array}{c}\text { SWIC-single } \\
\text { EEP }\end{array}$ & $\begin{array}{c}\text { PSWIC } \\
\text { UEP }\end{array}$ & $\begin{array}{c}\text { HSWIC } \\
\text { EEP }\end{array}$ & $\begin{array}{c}\text { Sherwood [9] } \\
\text { EEP }\end{array}$ & $\begin{array}{c}\text { Man [11] } \\
\text { UEP }\end{array}$ \\
\hline \hline 0.25 & 33.04 & 33.13 & 32.80 & $\mathbf{3 3 . 1 6}$ & 31.98 \\
\hline 0.5 & 36.23 & $\mathbf{3 6 . 2 8}$ & 36.02 & 36.25 & 35.08 \\
\hline 1.00 & 39.39 & $\mathbf{3 9 . 4 5}$ & 39.29 & 39.34 & N/A \\
\hline
\end{tabular}

ploy the SPIHT entropy coder. The method in [9] applies EEP to the SPIHT stream whereas the methods in [11], [14] apply UEP. The results are reported in Tables III and IV. Reconstructed images for various channel BERs and rates are shown in Fig. 7. Ten thousand MSE values were averaged and the outcome was converted to PSNR for calculating the entries in the tables. As seen, for low BERs $(\approx 0.001)$ the performance of all coders appear to be equivalent apart from the multiple-layer SWIC whose performance suffers due to the layer header information. Since at low BERs the transmission of puncturing matrix identifiers in multilayer coding schemes requires nonnegligible rate, UEP was used only with the PSWIC algorithm. For higher BERs $(\approx 0.01)$, the performance of the coders proposed here is clearly superior to that in [9] and competitive with that in [14]. This is also demonstrated in Fig. 8. In Fig. 9, detailed results for a large number of executions are shown.

The HSWIC results in Table III are intended to demonstrate the impact of the layer headers on the performance of the SWIC and PSWIC methods. The HSWIC results demonstrate that, for 


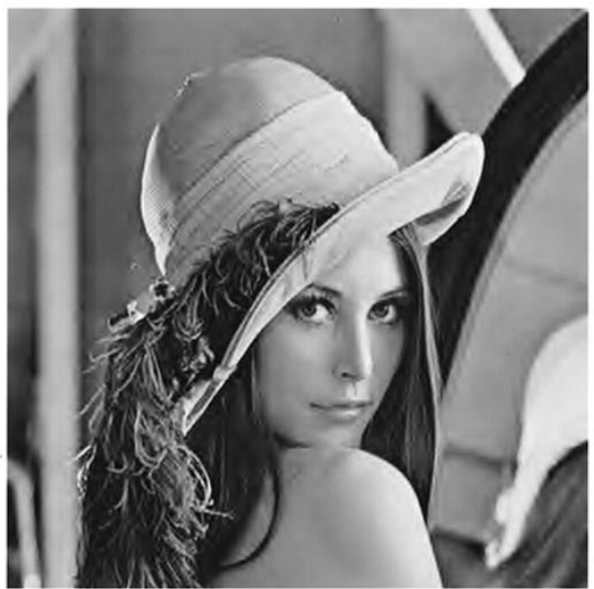

(a)

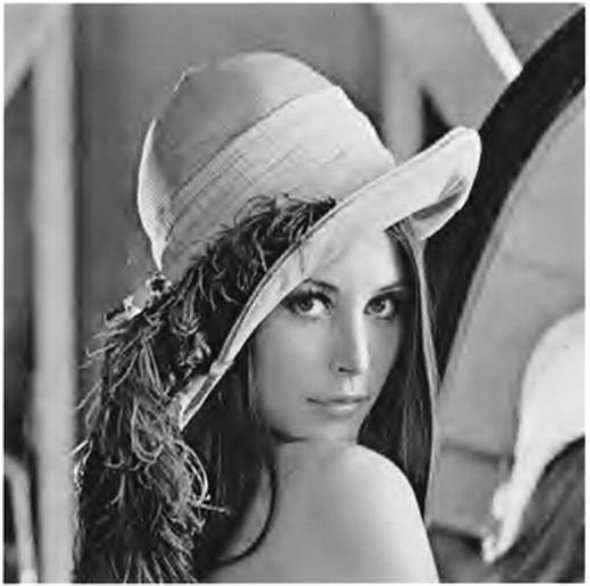

(c)

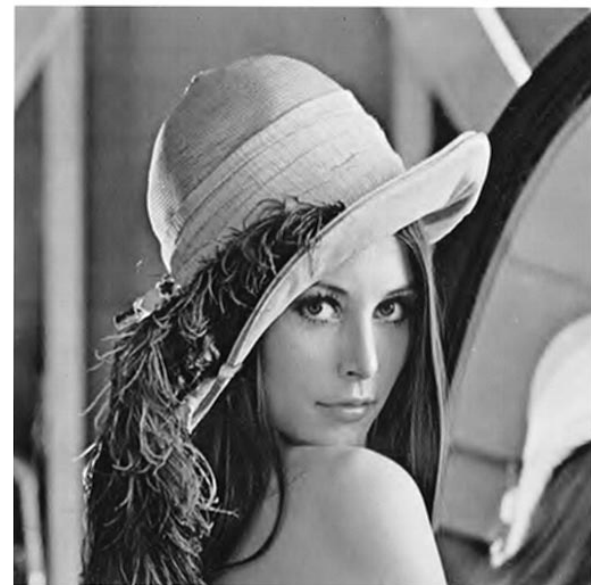

(b)

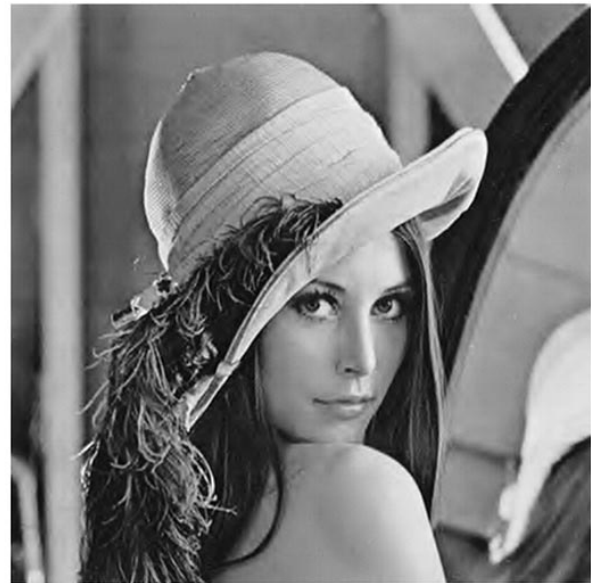

(d)

Fig. 7. Reconstructed "Lenna" when transmitted over noisy channels using the PSWIC algorithm: (a) 0.25 bpp and BER=0.001 (33.13 dB), (b) 0.5 bpp and $\mathrm{BER}=0.001(36.28 \mathrm{~dB}),(\mathrm{c}) 0.25 \mathrm{bpp}$ and $\mathrm{BER}=0.01(32.30 \mathrm{~dB}),(\mathrm{d}) 0.5 \mathrm{bpp}$ and $\mathrm{BER}=0.01(35.39 \mathrm{~dB})$.

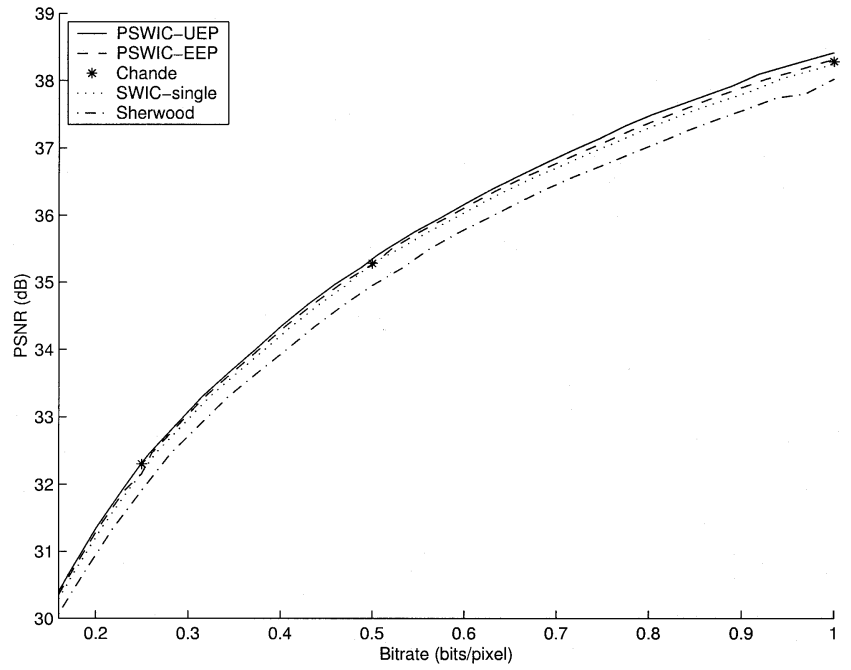

Fig. 8. Progressive transmission of images over a noisy channel with BER= 0.01 .

low bitrates, the employment of a predefined scan order saves a significant amount of rate which would otherwise be used for transmitting layer headers. This is clearly reflected in the results of Table III where a significant performance gain is shown in comparison to the multilayer SWIC results.

Another important feature of the multilayer coders (multilayer SWIC, PSWIC, HSWIC) is the fact that even in the case of channel mismatch (i.e., if the channel is noiser than originally estimated) the performance of our coder degrades gracefully with the increase of the bit error probability. Conventional robust coders stop at the occurrence of the first uncorrectable error resulting in significant abrupt decrease in performance due to the channel mismatch. However, for the multilayer SWIC coder, the reduction in the performance due to mismatch is much smaller. A comparison of our SWIC method, the SPIHT-based method in [9] and the method in [17] is demonstrated in Tables V, VI. We have used our own simulations of the methods in [9], [18] for obtaining performance results. The comparison is in terms of average MSE converted to PSNR over ten thousand independent simulations using the "Lenna" image. As expected, in cases where the channel is actually worse than estimated, the performance gap between our method and the method in [9] gets wider in favor of our coder. Moreover, the image quality at 1 bpp with the multilayer SWIC coder optimized for $\mathrm{BER}=0.01$ when the actual BER is equal to 0.02 is at least $1.5-2.0 \mathrm{~dB}$ above the quality achieved by the coder by Banister et al. in [18] 


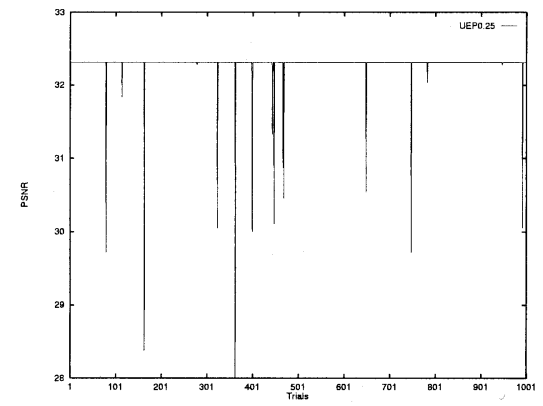

(a)

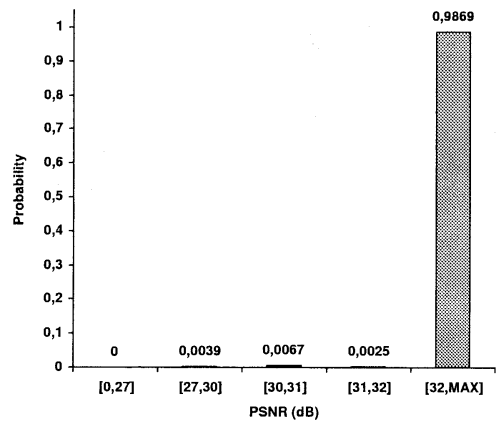

(d)

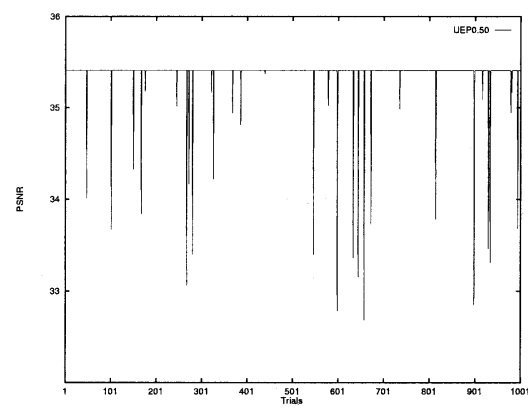

(b)

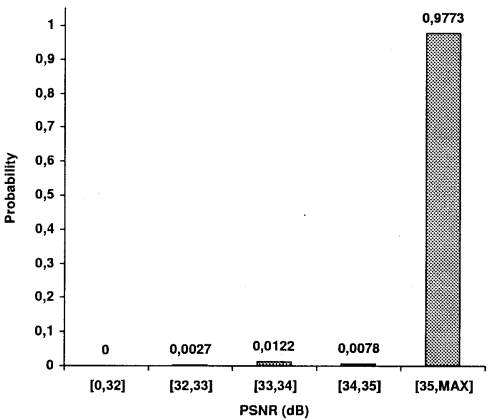

(e)

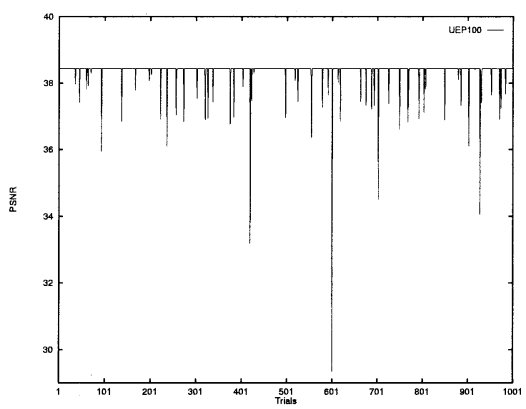

(c)

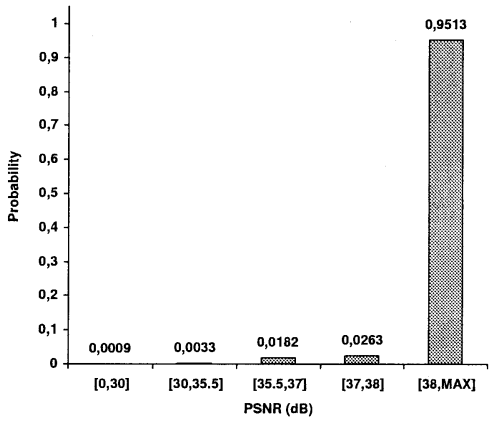

(f)

Fig. 9. Transmission of "Lenna" using PSWIC over a channel with BER $=0.01$. PSNR values for 1000 executions are shown. (a) Transmission at 0.25 bits/pixel. (b) Transmission at 0.50 bits/pixel. (c) Transmission at 1.00 bits/pixel. The corresponding distributions of PSNR values are shown in (d), (e), (f) respectively.

TABLE V

COMPARISON OF THE PROPOSED UEP-BASED SWIC SCHEME IN THE CASE OF ChanNEl Mismatch. OPtIMIZATION BER $=0.01$. ACtUAl BER $=0.02$

\begin{tabular}{c|c|c|c}
\hline Bitrate & SWIC & Sherwood [9] & Banister [18] \\
\hline 0.5 & 34.32 & 33.73 & 31.22 \\
\hline 0.75 & 35.53 & 35.28 & 31.59 \\
\hline 1.00 & 36.19 & 35.80 & 34.74 \\
\hline
\end{tabular}

TABLE VI

COMPARISON OF THE PRoposed UEP-BASED SWIC SCHEME IN THE CASE OF CHANNEL MisMatch. OPTIMIZATION BER $=0.01$. ACTUAL BER $=0.03$

\begin{tabular}{c|c|c|c}
\hline Bitrate & SWIC & Sherwood [9] & Banister [18] \\
\hline 0.5 & 26.22 & 26.22 & 24.24 \\
\hline 0.75 & 26.70 & 26.49 & 24.31 \\
\hline 1.00 & 29.82 & 26.54 & 30.23 \\
\hline
\end{tabular}

with an identical mismatch. ${ }^{2}$ This difference in performance becomes more notable if one considers that the coder in [18] employs EBCOT source coding (better than SPIHT and the source coders used in the present paper) and more powerful (and more complicated as well) turbo codes [35]. It is noted that the jump in improvement from 0.75 to 1.00 bpp compared to the SWIC and Banister [18] results in Table VI is due to the mismatch conditions which render the function of the distortion with respect to channel rate a largely not convex function.

${ }^{2}$ The authors in [18] report results in terms of mean PSNR rather than mean MSE converted to PSNR. In this work, we compare in terms of mean MSE converted to PSNR. The experimental results demonstrate that our method is superior. If, however, we compared on the basis of mean PSNR, the performance gap would be even wider in favor of our coder.
TABLE VII

STANDARD DEVIATION OF THE RECONSTRUCTION QUALITY (PSNR) FOR 10000 SIMULATIONS FOR THE TRANSMISSION OF IMAGES OVER $\mathrm{BSC}$ WITH BER $=0.01$

\begin{tabular}{c|c|c|c|c}
\hline & \multicolumn{2}{|c|}{ SWIC } & PSWIC & HSWIC \\
\cline { 2 - 3 } & single & multiple & UEP & UEP \\
\hline 0.25 & 0.289 & 0.05 & 0.319 & 0.066 \\
\hline 0.5 & 0.387 & 0.171 & 0.321 & 0.241 \\
\hline 1.00 & 1.461 & 0.349 & 0.349 & 0.440 \\
\hline
\end{tabular}

For the further evaluation of the reliability of our coders, the standard deviation of the PSNR values between the original and the reconstructed images was calculated for a large number of simulations. Results are reported in Table VII. As seen, the multiple-layer robust coders demonstrate the best behavior since the achieved reconstruction quality varies negligibly even when uncorrectable errors occur. However, when excessive numbers of layers are used, this comes at the cost of reduced average performance due to the need for transmission of a large number of layer headers. The PSWIC coder, which uses a moderate number of layers, appears to be a good compromise between reliability and performance.

Since our source coders perform approximately as well as (and often a little worse than) the SPIHT coder, our superior overall coding results can be primarily attributed to the organization of the bitstream in such a way that enables error localization and decoding beyond the point of an uncorrectable error. This feature alone makes the EEP-based versions of our coders perform better than state-of-the-art coders based on UEP. Additionally, the careful allocation of protection among layers makes the UEP variants of the proposed scheme even more efficient. 
The application of turbo codes to our coders would improve our results. However, since the optimization of the application of turbo codes would require the calculation of the optimal packet length along with several other adaptations, we do not further investigate it in this work.

Taking into consideration the experimental results, we reach the following conclusions.

- For channels with low BERs, e.g., $\epsilon=0.001$ or lower, coding of information as one layer is competitive to the coding using a moderate number of individual layers since the gain achieved by the enhanced capabilities of a coder with partitioned information is cancelled out by the side information required when such techniques are employed.

- For channels with high BERs, e.g., $\epsilon=0.01$ or higher, the coding of layers into independent streams appears to be preferable because of the ability of such streams to discard corrupted layers and decode the stream beyond the first uncorrectable error.

- In cases of channel mismatch, the performance of the independent layer coders degrades gracefully whereas in the case of coding in a single layer the decoding procedure may deteriorate badly.

- The bitstreams composed of independent layers appear to be generally more reliable for applications of image transmission over noisy channels since in the case of uncorrectable errors they are able to achieve decent reconstruction quality.

\section{CONCLUSION}

Novel joint source/channel coding schemes were proposed for the transmission of images over noisy channels. The proposed schemes are based on source coders which output a stream very suitable for robust transmission. Channel coding is applied on the layers of the source bitstream according to their importance. A blockwise optimization algorithm for the efficient unequal error protection of the embedded stream was also proposed. The resulting system was shown to deal very effectively with random errors. The proposed techniques can be easily adapted to the JPEG2000 standard which employs block-based coding strategies.

Application of more sophisticated channel coding techniques such as Reed-Solomon or turbo codes, would enable the application of our coders to the transmission of images over fading channels. This will be the subject of future research.

\section{APPENDIX}

PUnCTURING MATRICES USED FOR PUNCTURING THE OUTPUT OF THE CONVOLUTIONAL ENCODER

$$
\begin{gathered}
{\left[\begin{array}{ccccccc}
1 & 1 & 1 & 1 & 1 & 1 & 1 \\
1 & 0 & 0 & 0 & 0 & 0 & 0 \\
0 & 0 & 0 & 0 & 0 & 0 & 0 \\
0 & 0 & 0 & 0 & 0 & 0 & 0
\end{array}\right]\left[\begin{array}{ccccccc}
1 & 1 & 1 & 1 & 1 & 1 & 1 \\
1 & 0 & 0 & 0 & 1 & 0 & 0 \\
0 & 0 & 0 & 0 & 0 & 0 & 0 \\
0 & 0 & 0 & 0 & 0 & 0 & 0
\end{array}\right]} \\
\text { Rate } \frac{8}{9} .
\end{gathered}
$$

$$
\begin{aligned}
& {\left[\begin{array}{lllllll}
1 & 1 & 1 & 1 & 1 & 1 & 1 \\
1 & 0 & 1 & 0 & 1 & 0 & 0 \\
0 & 0 & 0 & 0 & 0 & 0 & 0 \\
0 & 0 & 0 & 0 & 0 & 0 & 0
\end{array}\right]\left[\begin{array}{lllllll}
1 & 1 & 1 & 1 & 1 & 1 & 1 \\
1 & 0 & 1 & 0 & 1 & 0 & 1 \\
0 & 0 & 0 & 0 & 0 & 0 & 0 \\
0 & 0 & 0 & 0 & 0 & 0 & 0
\end{array}\right]} \\
& \text { Rate } \frac{8}{11} \quad \text { Rate } \frac{8}{12} \\
& {\left[\begin{array}{llllllllllllllll}
1 & 1 & 1 & 1 & 1 & 1 & 1 & 1 & 1 & 1 & 1 & 1 & 1 & 1 & 1 & 1 \\
1 & 0 & 0 & 0 & 0 & 0 & 0 & 0 & 0 & 0 & 0 & 0 & 0 & 0 & 0 & 0 \\
0 & 0 & 0 & 0 & 0 & 0 & 0 & 0 & 0 & 0 & 0 & 0 & 0 & 0 & 0 & 0 \\
0 & 0 & 0 & 0 & 0 & 0 & 0 & 0 & 0 & 0 & 0 & 0 & 0 & 0 & 0 & 0
\end{array}\right]} \\
& \text { Rate } \frac{16}{17} \\
& {\left[\begin{array}{llllllllllllllll}
1 & 1 & 1 & 1 & 1 & 1 & 1 & 1 & 1 & 1 & 1 & 1 & 1 & 1 & 1 & 1 \\
1 & 0 & 0 & 0 & 1 & 0 & 0 & 0 & 1 & 0 & 0 & 0 & 0 & 0 & 0 & 0
\end{array}\right]} \\
& \begin{array}{llllllllllllllll}
1 & 0 & 0 & 0 & 1 & 0 & 0 & 0 & 1 & 0 & 0 & 0 & 0 & 0 & 0 & 0
\end{array} \\
& \begin{array}{llllllllllllllll}
0 & 0 & 0 & 0 & 0 & 0 & 0 & 0 & 0 & 0 & 0 & 0 & 0 & 0 & 0 & 0 \\
0 & 0 & 0 & 0 & 0 & 0 & 0 & 0 & 0 & 0 & 0 & 0 & 0 & 0 & 0 & 0
\end{array} \\
& {\left[\begin{array}{llllllllllllllll}
0 & 0 & 0 & 0 & 0 & 0 & 0 & 0 & 0 & 0 & 0 & 0 & 0 & 0 & 0 & 0
\end{array}\right]} \\
& \text { Rate } \frac{16}{19} \\
& {\left[\begin{array}{llllllllllllllll}
1 & 1 & 1 & 1 & 1 & 1 & 1 & 1 & 1 & 1 & 1 & 1 & 1 & 1 & 1 & 1 \\
1 & 0 & 1 & 0 & 1 & 0 & 0 & 0 & 1 & 0 & 0 & 0 & 1 & 0 & 0 & 0 \\
0 & 0 & 0 & 0 & 0 & 0 & 0 & 0 & 0 & 0 & 0 & 0 & 0 & 0 & 0 & 0 \\
0 & 0 & 0 & 0 & 0 & 0 & 0 & 0 & 0 & 0 & 0 & 0 & 0 & 0 & 0 & 0
\end{array}\right]} \\
& {\left[\begin{array}{llllllllllllllll}
1 & 1 & 1 & 1 & 1 & 1 & 1 & 1 & 1 & 1 & 1 & 1 & 1 & 1 & 1 & 1 \\
1 & 0 & 1 & 0 & 1 & 0 & 1 & 0 & 1 & 0 & 1 & 0 & 1 & 0 & 0 & 0 \\
0 & 0 & 0 & 0 & 0 & 0 & 0 & 0 & 0 & 0 & 0 & 0 & 0 & 0 & 0 & 0 \\
0 & 0 & 0 & 0 & 0 & 0 & 0 & 0 & 0 & 0 & 0 & 0 & 0 & 0 & 0 & 0
\end{array}\right]} \\
& \text { Rate } \frac{16}{23}
\end{aligned}
$$

\section{REFERENCES}

[1] N. V. Boulgouris, N. Thomos, and M. G. Strintzis, "Image transmission using error-resilient wavelet coding and forward error correction," in Proc. IEEE Int. Conf. Image Processing, vol. 3, Rochester, NY, Sept. 2002, pp. 549-552.

[2] A. Said and W. A. Pearlman, "A new fast and efficient image codec based on set partitioning in hierarchical trees," IEEE Trans. Circuits Syst. Video Technol., vol. 6, pp. 243-250, June 1996.

[3] D. Taubman, "High performance scalable image compression with EBCOT," IEEE Trans. Image Processing, vol. 9, pp. 1158-1170, July 2000.

[4] N. V. Boulgouris, D. Tzovaras, and M. G. Strintzis, "Lossless image compression based on optimal prediction, adaptive lifting and conditional arithmetic coding," IEEE Trans. Image Processing, vol. 10, pp. 1-14, Jan. 2001.

[5] K. Shen and E. J. Delp, "Wavelet based rate scalable video compression," IEEE Trans. Circuits Syst. Video Technol., vol. 9, pp. 109-122, Feb. 1999.

[6] D. Tzovaras and M. G. Strintzis, "Motion and disparity field estimation using rate-distortion optimization," IEEE Trans. Circuits Syst. Video Technol., vol. 8, pp. 281-290, Apr. 1998.

[7] C. E. Shannon, "Coding theorems for a discrete source with a fidelity criterion," in IRE Nat. Convention Record, Part 4, 1959, pp. 142-163.

[8] Y. Yang, S. Wenger, J. Wen, and A. K. Katsaggelos, "Error resilient video coding techniques," IEEE Signal Processing Mag., vol. 17, pp. 61-82, July 2000.

[9] G. Sherwood and K. Zeger, "Progressive image coding on noisy channels," IEEE Signal Processing Lett., vol. 4, pp. 189-191, July 1997.

[10] —, "Error protection for progressive image transmission over memoryless and fading channels," IEEE Trans. Commun., vol. 46, pp. 1555-1559, Dec. 1998.

[11] H. Man, F. Kossentini, and M. J. Smith, "A family of efficient and channel error resilient wavelet/subband image coders," IEEE Trans. Circuits Syst. Video Technol., vol. 9, pp. 95-108, Feb. 1999.

[12] N. Tanabe and N. Farvardin, "Subband image coding using entropy-coded quantization over noisy channels," IEEE J. Select. Areas Commun., vol. 10, pp. 926-943, June 1992.

[13] G. Davis and J. Danskin, "Joint source and channel coding for image transmission over lossy packet networks," in Proc. SPIE, vol. 2847, Apr. 1996, pp. 376-387. 
[14] V. Chande and N. Farvardin, "Progressive transmission of images over memoryless noisy channels," IEEE J. Select. Areas Commun., vol. 18 , pp. 850-860, June 2000.

[15] A. E. Mohr, E. A. Riskin, and R. E. Ladner, "Unequal loss protection: graceful degradation of image quality over packet erasure channels through forward error correction," IEEE J. Select. Areas Commun., vol. 18 , pp. 819-828, June 2000.

[16] A. Albanese, J. Bloemer, J. Edmonds, M. Luby, and M. Sudan, "Priority encoding transmission," IEEE Trans. Inform. Theory, vol. 42, pp. 1737-1744, Nov. 96.

[17] A. A. Alatan, M. Zhao, and A. N. Akansu, "Unequal error protection of SPIHT encoded image bitstreams," IEEE J. Select. Areas Commun., vol. 18, pp. 814-818, June 2000.

[18] B. A. Banister, B. Belzer, and T. R. Fisher, "Robust image transmission using JPEG2000 and turbo codes," IEEE Signal Processing Lett., vol. 9, pp. 117-119, Apr. 2002.

[19] C. Christopoulos, A. Skodras, and T. Ebrahimi, "The JPEG2000 still image coding system: an overview," IEEE Trans. Consumer Electron., vol. 46, pp. 1103-1127, Nov. 2000.

[20] J. M. Shapiro, "Embedded image coding using zerotrees of wavelet coefficients," IEEE Trans. Signal Processing, vol. 41, pp. 3445-3462, Dec. 1993.

[21] I. H. Witten, R. M. Neal, and J. G. Cleary, "Arithmetic coding for data compression," Commun. ACM, vol. 30, pp. 520-540, June 1987.

[22] E. Ordentlich, M. Weinberger, and G. Seroussi, "A low-complexity modeling approach for embedded coding of wavelet coefficients," in Proc. IEEE Data Compression Conf., Snowbird, UT, Mar. 1998, pp. 218-227.

[23] J. Li and S. Lei, "An embedded still image coder with rate-distortion optimization," IEEE Trans. Image Processing, vol. 8, pp. 913-924, July 1999.

[24] B. Usevitch, "Optimal bit allocation for biorthogonal wavelet coding," in Proc. DCC Data Compression Conf., Snowbird, UT, 1996, pp. 387-395.

[25] A. Bilgin, P. J. Sementilli, F. Sheng, and M. W. Marcellin, "Scalable image coding using reversible integer wavelet transforms," IEEE Trans. Image Processing, vol. 9, pp. 1972-1977, Nov. 2000.

[26] C. Chrysafis, "Wavelet image compression rate distortion optimizations and complexity reductions," Ph.D. dissertation, Univ. Southern California, Los Angeles, 1999.

[27] J. Hagenauer, "Rate-compatible punctured convolutional codes (RCPC Codes) and their applications," IEEE Trans. Commun., vol. 36, pp. 389-400, Apr. 1989.

[28] S. Lin and D. J. Costello, Error Control Coding: Fundamentals and Applications. Englewood Cliffs, NJ: Prentice-Hall, 1982.

[29] N. Seshadri and C.-E. Sundberg, "List Viterbi decoding algorithm with applications," IEEE J. Select. Areas Commun., vol. 42, no. 2/3/4, pp. 313-323, Feb./Mar./Apr. 1994.

[30] I. Kozintsev, J. Chou, and K. Ramchandran, "Image transmission using arithmetic coding based continuous error detection," in Proc. IEEE DCC Data Compression Conf., Snowbird, UT, Mar. 1998, pp. 339-348.

[31] P. Moo and X. Wu, "Resynchronization properties of arithmetic coding," in Proc. IEEE Int. Conf. Image Processing, Kobe, Japan, Oct 1999, pp. $545-549$.

[32] A. Ortega and K. Ramchandran, "Rate-distortion methods for image and video compression," IEEE Signal Processing Mag., vol. 15, pp. 23-50, Nov. 1998.

[33] Y. Shoham and A. Gersho, "Efficient bit allocation for an arbitrary set of quantizers," IEEE Trans. Acoust., Speech, Signal Processing, vol. 36, pp. 1445-1453, Sept. 1988.
[34] G. Castagnoli, J. Ganz, and P. Graber, "Optimum cyclic redundancycheck codes with 16-Bit redundancy," IEEE Trans. Commun., vol. 38 , pp. 111-114, Jan. 1990

[35] C. Berrou and A. Glavieux, "Near optimum error correcting coding and decoding: Turbo codes," IEEE Trans. Commun., vol. 44, pp. 1261-1271, Oct. 1996.

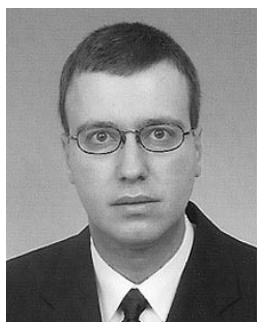

Nikolaos V. Boulgouris received the Diploma and the Ph.D. degrees from the University of Thessaloniki, Thessaloniki, Greece, in 1997 and 2002 , respectively.

Since September 2003, he has been a Post-Doctoral Fellow with the Department of Electrical and Computer Engineering, University of Toronto, Toronto, ON, Canada. Formerly, he was a researcher with the Informatics and Telematics Institute, Thessaloniki, Greece. During his graduate studies, he held several research and teaching assistantship positions. Since 1997, he has participated in research projects in the areas of image/video communications, pattern recognition, multimedia security, and content-based indexing and retrieval

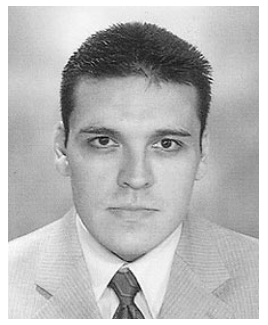

Nikolaos Thomos (S'02) was born in Cologne, Germany, in 1977. He received the Diploma in electrical and computer engineering from Aristotle University of Thessaloniki, Thessaloniki, Greece, in 2000. He is currently working toward the Ph.D. degree at the same university.

$\mathrm{He}$ holds research and teaching assistantship positions in the Electrical and Computer Engineering Department, Aristotle University of Thessaloniki. $\mathrm{He}$ is also a Graduate Research Assistant with the Informatics and Telematics Institute, Thessaloniki, Greece. His research interests include image and video coding/transmission, multimedia networking, wavelets, and digital filters.

Mr Thomos is a member of the Technical Chamber of Greece,

Michael G. Strintzis (S'68-M'70-SM'80) received the Diploma in electrical engineering from the National Technical University of Athens, Athens, Greece in 1967, and the M.A. and Ph.D. degrees in electrical engineering from Princeton University, Princeton, NJ, in 1969 and 1970, respectively.

He then joined the Electrical Engineering Department, University of Pittsburgh, Pittsburgh, PA, where he served as Assistant (1970-1976) and Associate (1976-1980) Professor. Since 1980, he has been a Professor of Electrical and Computer Engineering at the University of Thessaloniki, Thessaloniki, Greece, and since 1999 Director of the Informatics and Telematics Research Institute, Thessaloniki. His current research interests include two- and three-dimensional image coding, image processing, biomedical signal and image processing, and DVD and Internet data authentication and copy protection.

Dr. Strintzis was awarded one of the Centennial Medals of the IEEE in 1984 Since 1999, he has served as an Associate Editor of the IEEE TRANSACTIONS ON CIRCUITS AND SYSTEMS FOR VIDEO TECHNOLOGY. 